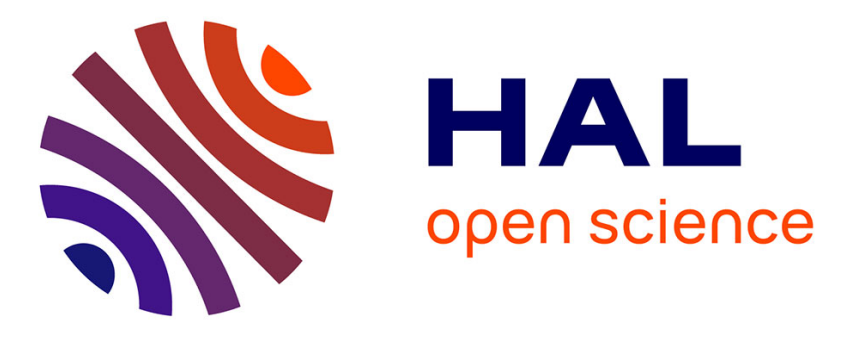

\title{
Synaptic Coupling Between Two Electronic Neurons
}

Sabir Jacquir, Stéphane Binczak, Jean-Marie Bilbault, Victor B. Kasantsev, V.I. Nekorkin

\section{To cite this version:}

Sabir Jacquir, Stéphane Binczak, Jean-Marie Bilbault, Victor B. Kasantsev, V.I. Nekorkin. Synaptic Coupling Between Two Electronic Neurons. Nonlinear Dynamics, 2006, 44, pp.29-36. 10.1007/s11071006-1932-6 . hal-00583790

\section{HAL Id: hal-00583790 https://u-bourgogne.hal.science/hal-00583790}

Submitted on 6 Apr 2011

HAL is a multi-disciplinary open access archive for the deposit and dissemination of scientific research documents, whether they are published or not. The documents may come from teaching and research institutions in France or abroad, or from public or private research centers.
L'archive ouverte pluridisciplinaire HAL, est destinée au dépôt et à la diffusion de documents scientifiques de niveau recherche, publiés ou non, émanant des établissements d'enseignement et de recherche français ou étrangers, des laboratoires publics ou privés. 


\title{
Synaptic Coupling Between Two Electronic Neurons
}

\author{
SABIR JACQUIR ${ }^{1}$, STÉPHANE BINCZAK ${ }^{1, *}$, JEAN-MARIE BILBAULT ${ }^{1}$, \\ VIKTOR KAZANTSEV ${ }^{2}$, and VLADIMIR NEKORKIN ${ }^{2}$ \\ ${ }^{1}$ Laboratoire LE2I UMR CNRS 5158, Aile des Sciences de l'Ingénieur, Université de Bourgogne, BP 47870, Dijon Cedex, \\ France; ${ }^{2}$ Institute of Applied Physics of RAS, 46 Uljanov str., 603950 Nizhny Novgorod, Russia; \\ *Author for correspondence (e-mail: stbinc@u-bourgogne.fr; fax: +33-380395910)
}

(Received: 30 August 2004; accepted: 17 March 2005)

\begin{abstract}
An electrical circuit is proposed to realize an unidirectional coupling between two cells, mimicking chemical synaptic coupling. Each cell represents the FitzHugh-Nagumo (FHN) model of neuron with a modified exitability (MFHN). We present experimental results on frequency doublings and on the chaotic dynamics depending on the coupling strength in a master-slave configuration. In all experiments, we stress the influence of the coupling strength on the control of the slave neuron.
\end{abstract}

Key words: bifurcation, electronic neuron, FitzHugh-Nagumo model, synaptic coupling

Abbreviations: MFHN - modified FitzHugh-Nagumo

\section{Introduction}

Although the differential equations are used to model the nerve membrane, we suggest describing the overall activity of neurons by one of these models. Moreover, we propose an experimental electronic implementation of it. Indeed in the literature, some electronic neurons, such as the Nagumo's lattice [1] and the Neuristor device [2], have been realized. In the first section of this paper, the FitzHughNagumo (FHN) equation with modified excitability has been used to conceive the electronic MFHN neuron [3]. Its experimental bifurcation curves in the dimensionless plane $(\eta, \varepsilon)$ is given. This MFHN circuit leads to complex dynamics of traveling waves $[4,5]$ emerging from saddle homoclinic loop bifurcations. In the second section, we use the MFHN circuit as a basic cell to realize a master-slave configuration. Two cells are coupled in an unidirectional manner, which would correspond to two neurons coupled synaptically. After the presentation of the electronic circuit giving this coupling, we discuss the experimental conditions for which the master dynamics controls the excitability of the slave neuron leading to a shift of bifurcation curves, a variation of an eigen interspike frequency or a phenomenon of intermittency route to chaos.

\section{Experimental Description of One Cell}

\subsection{Electrical Circuit}

The nonlinear circuit, as sketched in Figure 1, can be described as follows: Part (A) is a parallel association of three different branches, two of them being resistive and commuted by silicium diodes $(V d=0.6 V)$, while the third is a negative resistor obtained with an operational amplifier. 


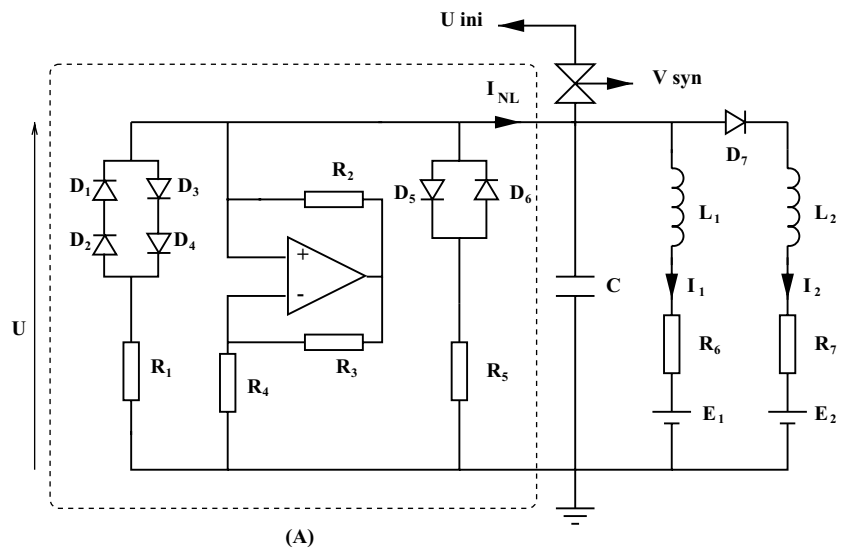

Figure 1. Diagram of the nonlinear circuit.

Due to diodes' commuting behavior, the resulting $I-V$ characteristic is nonlinear and can be modeled by a cubic polynomial function for an appropriate set of parameters so that

$$
I_{\mathrm{NL}}=f(U)=\frac{1}{R_{0}}\left[U-\frac{\gamma^{2} U^{3}}{3}\right]
$$

where $U$ and $I_{\mathrm{NL}}$ are respectively the voltage and the corresponding current. The parameters $R_{0}$ and $\gamma$ are obtained by a fitting approximation, e.g. by least mean square method. We obtain a good match between experimental results and Equation (1) by setting $R_{0}=1023 \Omega$ and $\gamma=1.138 \mathrm{~V}^{-1}$ [3]. This nonlinear resistor is in parallel with a capacitance and two branches in parallel including inductances, resistances and voltage sources, one of them being commuted by a silicium diode so that setting the conditions $\frac{R_{6}}{L_{1}}=\frac{R_{7}}{L_{2}}, E_{2}=-V d$, and using a piecewise linear $I-V$ description for diode $D_{7}, I_{2}=0$ if $U<0$. Therefore, using Kirchhoff's laws, the system of equations can be expressed in a normalized way by:

$$
\left\{\begin{array}{l}
\frac{d V}{d \tau}=\left[V-\frac{V^{3}}{3}\right]-W \\
\frac{d W}{d \tau}=\varepsilon[g(V)-W-\eta]
\end{array}\right.
$$

where $V=\gamma U$ and $W=\gamma R_{0}\left(I_{1}+I_{2}\right)$ correspond, in biological terms, to the membrane voltage and the recovery variable; $\tau=\frac{t}{R_{0} C}$ is a re-scaled time, $\varepsilon=\frac{R_{0} R_{6} C}{L_{1}}$ the recovery parameter and $\eta=\gamma \frac{R_{0}}{R_{6}} E_{1}$ a bifurcation parameter. $g(V)$ is a piecewise linear function, $g(V)=\alpha V$ if $V \leq 0$ and $g(V)=\beta V$ if $V>0$ where $\alpha=\frac{R_{0}}{R_{6}}$ and $\beta=\frac{L_{1}+L_{2}}{L_{2}} \frac{R_{0}}{R_{6}}$ control the shape and location of the W-nullcline [3].

\subsection{EXPERIMENTAL BifuRCATION CURVES OF MFHN CIRCUIT}

This section presents different dynamics of the MFHN neuron [3]. In the case, $\alpha=\beta=1$, the system corresponds to the standard FHN equation where nullcline can only intersect at a single equilibrium point leading to Andronov-Hopf bifurcations. In the general case $\alpha \neq \beta$, resolving Equation (2), three nullcline points are expected. The phase portrait is very similar to the one occurring from the modified Morris-Lecar Equations [6,7] proposed to model barnacle muscle fibres and pyramidal cells. 


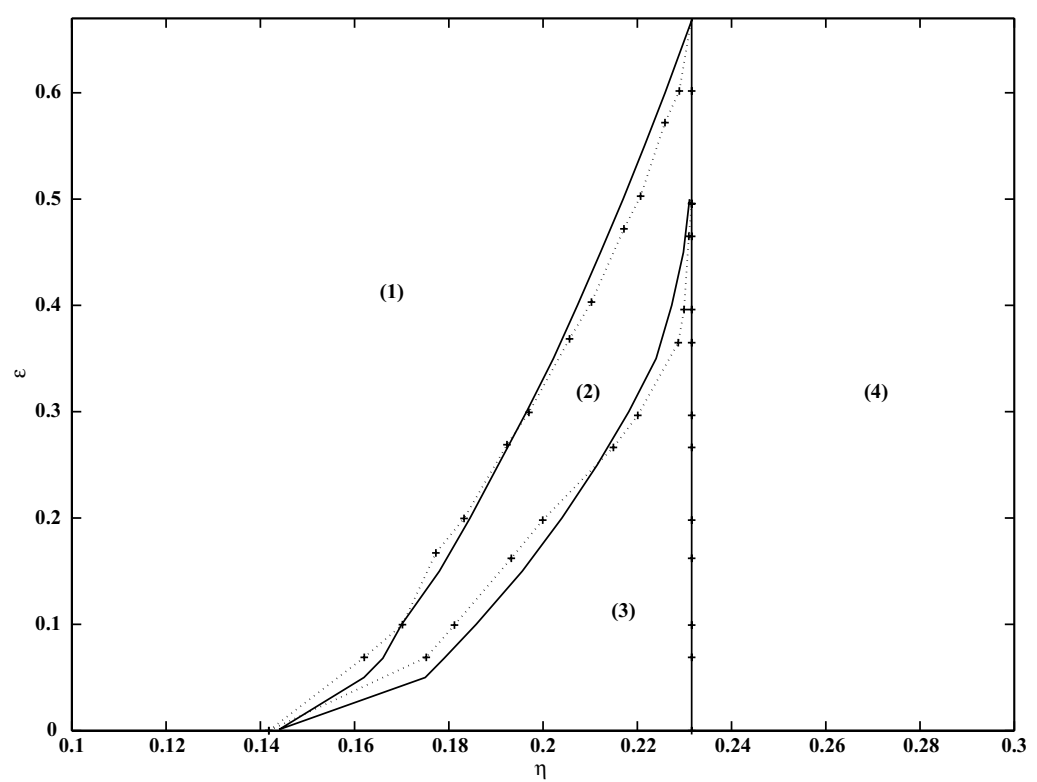

Figure 2. Comparison between experimental results ( + , linked by dot line) and numerical simulation (continuous line) in the dimensionless plane $(\eta, \varepsilon)$.

Considering the case $\alpha=0.506$ and $\beta=1.97$, an experimental and numerical simulation determination of the bifurcation curves in the parameter plane $(\eta, \varepsilon)$ is proposed in Figure 2.

Several results are to be distinguished depending on the location of the nullcline points and on initial conditions. In domain (1), two points are stable and unstable foci, while one point is a saddle, the system responds with an excitation pulse. Domain (2) corresponds to the bistability case characterized by the existence of a stable fixed point and a stable limit cycle that has appeared from a big homoclinic loop bifurcation. Then the model exhibits oscillations if the perturbation is large enough. In domain (3), the fixed point looses stability via a subcritical Andronov-Hopf bifurcation and only those oscillations occur in the model that are similar to the spiking train of pulses. Note that in the region (2), another saddle homoclinic loop bifurcation has taken place, leading to a small unstable limit cycle near the fixed point. Experiments have shown that, contrary to standard FHN, arbitrarily long interspike intervals can be found, as the two lower equilibrium points are merging. Finally, in domain (4), a single unstable fixed point exists, leading to oscillations; See [3] for more details. The existence of these domains has been confirmed with numerical simulations and stability analysis of Equation (2). In the following section, we study a specific case of coupling between two cells.

\section{Unidirectional Coupling of Two Cells}

The neurons communicate mainly between themselves through specialized devices called synapses via chemical messages. The chemical synapse transmits the impulse unidirectionally. Therefore, it is interesting to conceive and to realize an electrical circuit including the same features as the synaptical coupling. We present in Figure 3 the unidirectional coupling between two cells leading to a master-slave configuration, where $N_{i}(i=1,2)$ are described by the circuit of Figure 1 .

Let us introduce $D$, the coupling parameter (synaptic strength). Its circuit, as illustrated in Figure 4, includes an adder-inverter, an inverter and then a follower. 


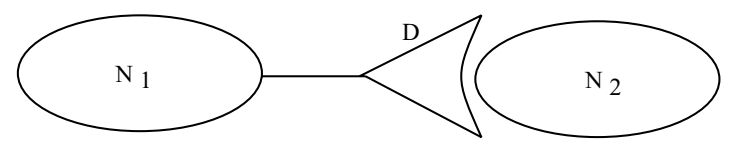

Figure 3. Coupling between two cells $N_{1}$ and $N_{2}$.

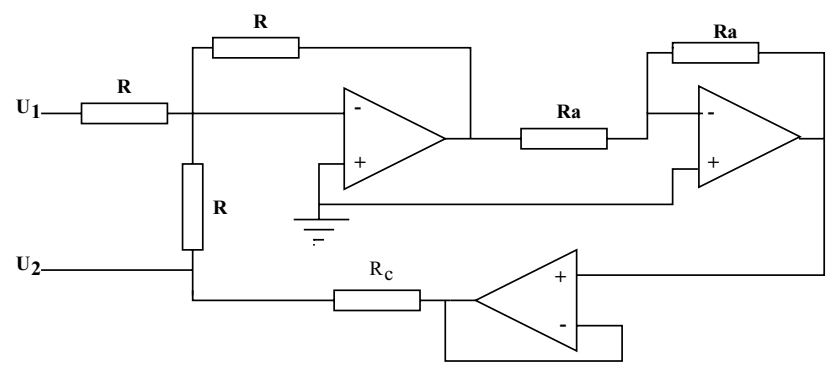

Figure 4. Unidirectional coupling circuit.

$U 1$ (resp. $U 2$ ) is the voltage capacitor of the cell $N 1$ (resp. N2). The value of the resistor $R$ is fixed to $100 \mathrm{k} \Omega$, which is large compared to the other components so that the current going though $2 R$ is negligible, $R_{a}=10 \mathrm{k} \Omega$, while $R_{c}$ is a detuning parameter, which allows us to control the coupling parameter value. Note also that the initial condition can be loaded in the neuron via an analogue commutator controlled by $V_{\text {syn }}$. Although it is usual to study a system with normalized variables, it is more convenient to describe electrical circuits with experimental variables. Therefore, we will keep both variables (as normalized $V_{1}$ and experimental $U_{1}$ ) in the following of this paper. Using Kirchoff's laws, the normalized equations corresponding to the coupling between two MFHN neurons in a master-slave configuration can be expressed by:

$$
\left\{\begin{array}{l}
\frac{d V_{1}}{\mathrm{~d} \tau}=\left[V_{1}-\frac{V_{1}^{3}}{3}\right]-W_{1} \\
\frac{d W_{1}}{\mathrm{~d} \tau}=\varepsilon_{1}\left[g\left(V_{1}\right)-W_{1}-\eta_{1}\right] \\
\frac{d V_{2}}{\mathrm{~d} \tau}=\left[V_{2}-\frac{V_{2}^{3}}{3}\right]-W_{2}+D V_{1} \\
\frac{d W_{2}}{d \tau}=\varepsilon_{2}\left[g\left(V_{2}\right)-W_{2}-\eta_{2}\right]
\end{array}\right.
$$

with $D=\frac{R_{0}}{R_{c}}$, and where the subscripts 1 and 2 indicate respectively the equations of the master and the slave neuron.

Therefore, the two neurons are coupled so that a part of current weighted by $D$ via $R$, and generated by $N_{1}$ is included in $N_{2}$. The two neurons are initially set to voltage $U_{1}$ ini and $U_{2}$ ini, due to the analogue commutators controlled by voltage $V_{\text {syn }}$. When the initial conditions are loaded, these commutators are switched off, while the two neurons are connected via a third commutator controlled by $\overline{V_{\text {syn }}}$. Note that the time delay between the two neurons has not been taken into account in this circuit, a master-slave configuration rendering it unnecessary. 


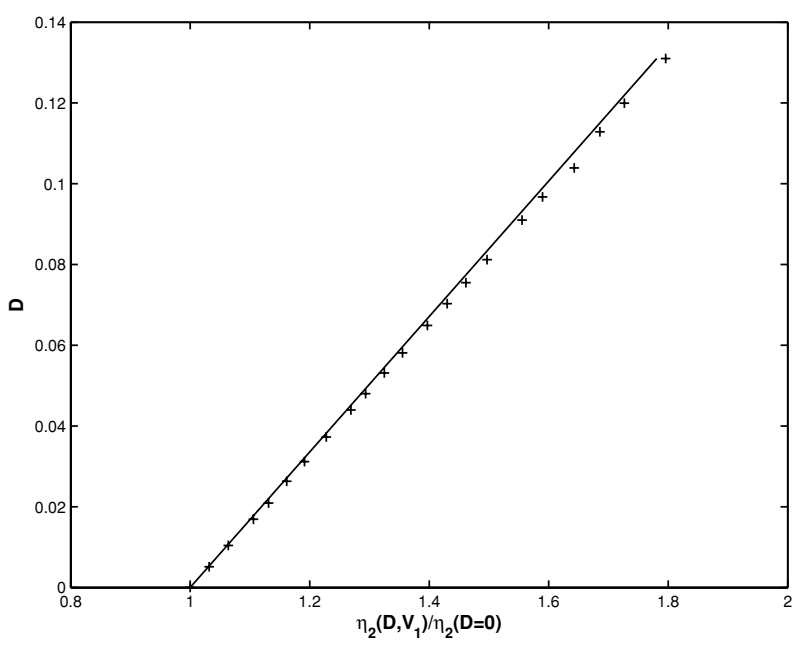

Figure 5. Shifted bifurcation curve of the slave neuron $N_{2}$ between domains (1) and (3). Parameters: master neuron $N_{1}: \alpha_{1}=$ $0.506, \beta_{1}=1.97, \epsilon_{1}=0.01, \eta_{1}=0.01$ leading to $V_{1}=-1.05$ (i.e. $U_{1}=-921 \mathrm{mV}$ ). Slave neuron $N_{2}: \alpha_{2}=0.506, \beta_{2}=1.97$, $\epsilon_{2}=0.01$. Comparison between Equation (4) (continuous line) and experimental results (+).

\subsection{The Master in a Resting State}

When the voltage is so that $V_{1}$ is constant (the cell $N_{1}$ is in a resting state); it is straightforward to show that the variable $W_{2}$ and the bifurcation parameter $\eta_{2}$ of the cell $N_{2}$ are given by:

$$
\left\{\begin{array}{l}
\eta_{2}\left(V_{1}\right)=\eta_{2}\left(V_{1}=0\right)+D V_{1} \\
W_{2}\left(V_{1}\right)=W_{2}\left(V_{1}=0\right)-D V_{1}
\end{array}\right.
$$

Therefore, it implies a modification of the excitability of the cell $N_{2}$ corresponding to a shift in the $(\eta, \varepsilon)$ plane illustrated in Figure 5. The initial conditions are so that, when $D=0$, the master neuron $N_{1}$ lies in domain (1), while the slave neuron $N_{2}$ is in domain (3) and generates a spiking train of pulses. When the unilateral coupling is increased and reaches a critical value, the neuron $N_{2}$ ceases to oscillate and stays in the resting state, meaning that the slave neuron has been moved from domain (3) to domain (1) of Figure 2. This ability of neuron $N_{1}$ to inhibit neuron $N_{2}$ corresponds to the shift predicted by Equation (4): As $V_{1}<0$, increasing $D$ implies to increase $\eta_{2}\left(D, V_{1}\right)$ and therefore the bifurcation curves of Figure 2 are translated along abscissa, while the value of $\eta_{2}$ defined by the electrical parameters of neuron $N_{2}$ has not been changed. This result suggests that, for a defined activity of a slave neuron, the strength of a unilateral coupling should be above a critical value to give to the master neuron the control on the slave neuron.

In Figure 5, experimental values $\left(D, \eta\left[D, V_{1}\right]\right)$ correspond to the shifted bifurcation curve between domains (1) and (3) of the neuron $N_{2}$ with $\varepsilon_{2}=0.01$ and when the master neuron $N_{1}$ lies in domain (1) in a resting state so that $V_{1}=-1.05$. Comparison shows a good match between experimental results $(+)$ and Equation (4) (continuous line), validating the unilateral coupling circuit.

\subsection{The Master in a SPIKING Regime}

In this section, we present some results when the master is in domain (2) and oscillates. As $V_{1}$ is varying in time, we cannot express a simple relationship between the parameters of neuron $N_{2}$ and $V_{1}$, as in 


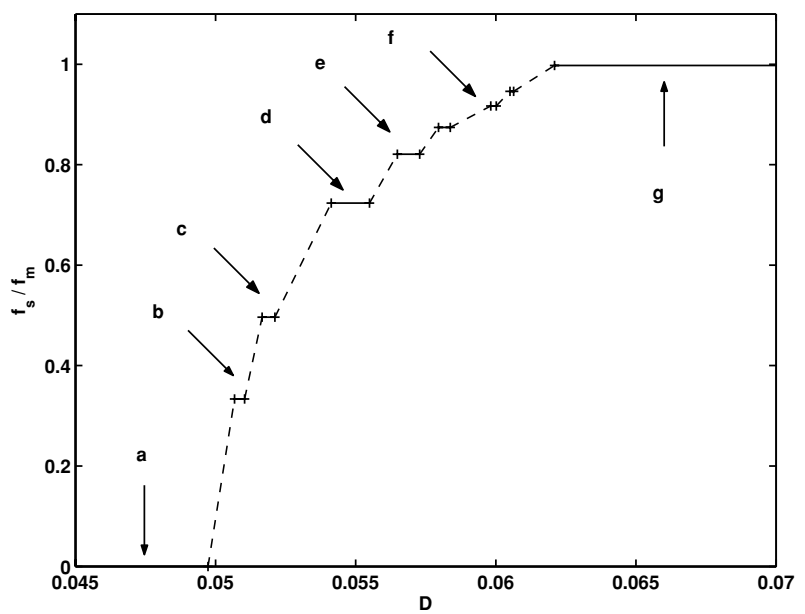

Figure 6. Normalized eigen interspike slave frequency $f_{\mathrm{s}}$ by interspike master frequency $f_{\mathrm{m}}$ versus $D$ with $C=0.001 \mathrm{nF}$, $\epsilon_{1}=\epsilon_{2}=0.002, \eta_{1}=0.199$ and $\eta_{2}=0.109$.

Equation (4). Nevertheless, oscillations of neurons $N_{1}$ let $V_{1}$ be alternatively positive and negative, which implies that the bifurcation curves of neurons are translated along the abscissa in the plane $(\eta, \epsilon)$ in a periodic manner (the position of saddle points of the cell $N_{2}$ is moved periodically). Thus, the slave neuron $N_{2}$ initially situated in the vicinity of a bifurcation curve may be able to cross sometimes this curve and develop a different dynamical behavior. Therefore, we have investigated the unilateral coupling influence on $N_{2}$, in the case when neuron $N_{1}$ is initially (i.e. when $D=0$ ) in domain (3) and oscillating, while the slave neuron $N_{2}$ is in domain (1).

According to the value of $D$, several different dynamical behaviors can be identified, as illustrated in Figures 6 and 7:

- In case (a), the coupling strength is small, leading to subthreshold oscillations of $f_{\mathrm{m}}$ frequency, which are not taken into account in Figure 6 but are observable in Figure 7.

- In cases (b)-(f), stable periodic oscillations appear whose eigen interspike frequency follows a devil's staircase-like curve. Only specific values of $f_{\mathrm{s}} / f_{\mathrm{m}}$ are obtainable. Increasing the coupling strength causes the period doubling in the slave cell, that is the period is multiplied by 2, 4, 8, 16, and so on.

- In case (g), $N_{2}$ is fully synchronized with $N_{1}$. The slave neuron oscillates in the same manner as the master.

The slave neuron shows also a chaotic sequence of spikes resulting with variable interspike intervals. This chaotic regime, corresponding to the dot lines in Figure 6, between the period-doubling plateaus is so that the interspike slave period is varying during the experiments. When the coupling parameter is gradually increased, we firstly proceed from a periodic spiking regime to a chaotic regime via a sequence of period-doubling bifurcations. Finally, it leads to the reappearance of periodic dynamics inserted in the chaotic zones.

The chaotic puffs disturb the periodic spiking regime. Increasing the coupling parameter causes the increase of the frequency disturbances and then the chaos dominates the regime in the slave cell $[8,9]$. An illustration of a chaotic signal is given in Figure 8 for $D=0.0535$. The corresponding probability of normalized interspike slave frequency $f_{\mathrm{s}} / f_{\mathrm{m}}$ is presented in Figure 9 . This figure shows that in a chaotic regime, the interspike frequencies are distributed widely in the range $\left[0,0.4 f_{\mathrm{m}}\right]$.

These experiments show that the unilateral coupling strength controls the slave neuron, from a silent to a chaotic dynamical behavior. 


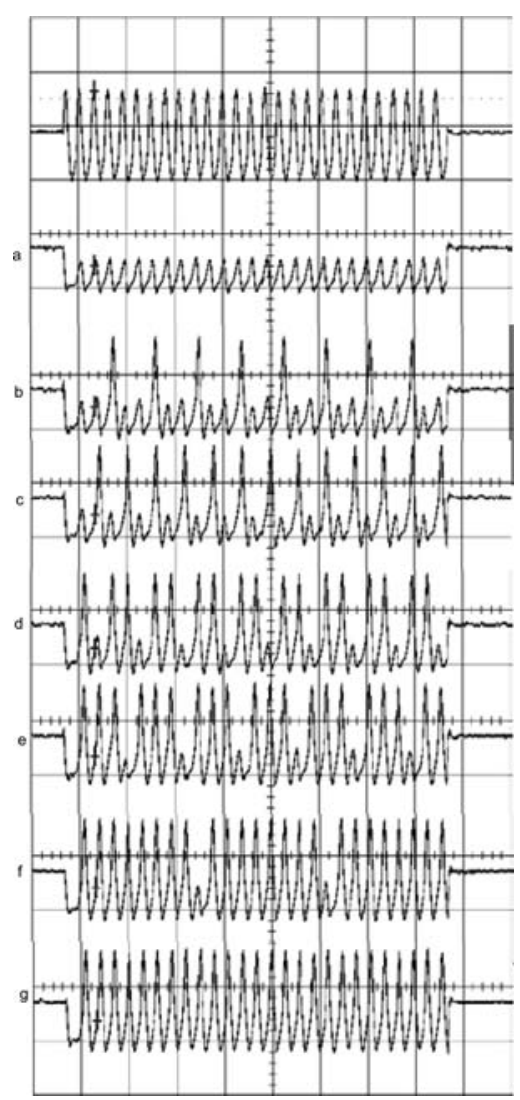

Figure 7. Temporal evolution of experimental voltage $U_{2}$ for different values of $D$ corresponding to cases (a)-(g) of Figure 6 . Voltage of the master neuron $N_{1}$ is shown on top. Abscissa: $0.1 \mathrm{~ms}$ per division; ordinate: $2 \mathrm{~V}$ per division.

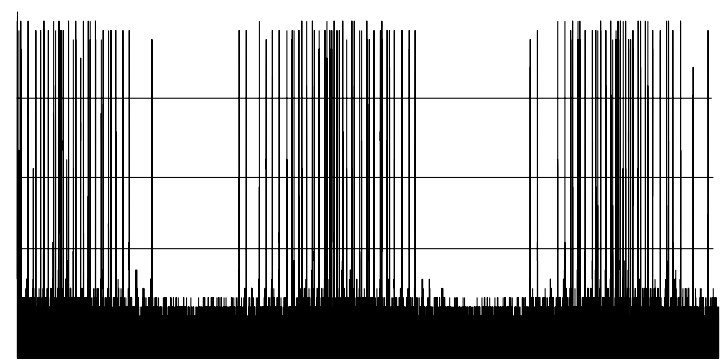

Figure 8 . Chaotic signal in the case where $D=0.0535$. Abscissa: $5 \mu$ s per division; ordinate: $2 \mathrm{~V}$ per division.

\section{Conclusion}

We have introduced an electrical circuit that allowed an unidirectional coupling without delay between two cells in a master-slave configuration. We have shown that the intervals between successive spikes can be chaotic and depend on the coupling strength. We suggest that this study can be helpful in understanding the different dynamics of potential propagation in brain cells. To complete this work, it would be of interest to study the bidirectional coupling corresponding to the electric synapse case and 


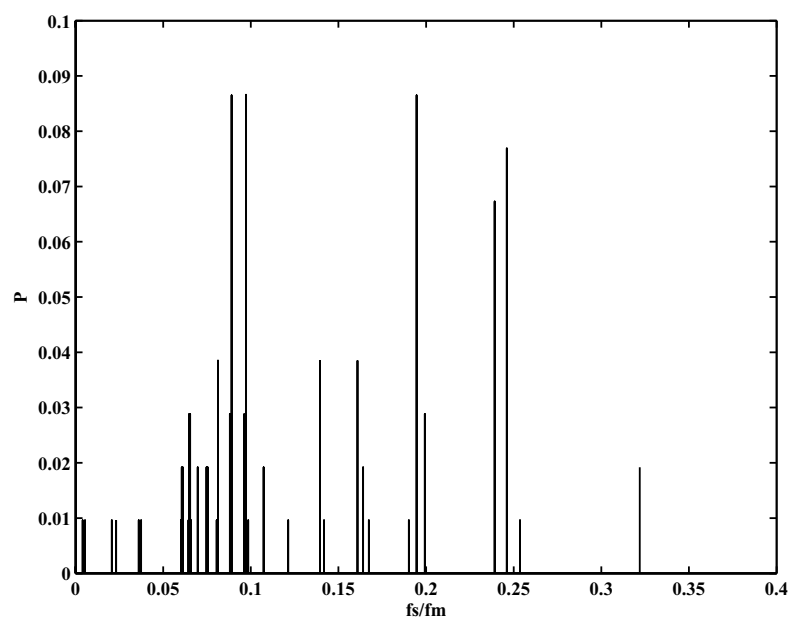

Figure 9. Normalized distribution of the interspike frequency of $N_{2}$ corresponding to the signal in Figure 8.

the influence of the size of the network on the fractal dimension of the information [10]. This nonlinear electrical circuit cell indeed gives the opportunity to realize a large-scale network.

\section{Acknowledgements}

This research has been supported by the Russian-French program of joint research (grant 04610PA), the Russian Foundation for Basic Research (grant 03-02-17135) and INTAS grant (YSF 2001-2/24).

\section{References}

1. Nagumo, J., Arimoto, S., and Yoshizawa, S., 'An active impulse transmission line simulating nerve axon', Proceedings of IRE 50, 1962, 2061-2070.

2. Scott, A. C., Neuroscience: A Mathematical Primer, Springer-Verlag, New York, 2002.

3. Binczak, S., Kazantsev, V. B., Nekorkin, V. I., Bilbault, J. M., 'Experimental study of bifurcations in modified FitzHughNagumo cell', Electronic Letter 39, 2003, 961-962.

4. Dawson, S. P., D'Angelo, M. V., and Pearson, J. E., 'Towards a global classification of excitable reaction-diffusion systems', Physics Letter A 265, 2000, 346-352.

5. Kazantsev, V. B., Nekorkin, V. I., Binczak, S., and Bilbault, J. M., 'Spiking patterns emerging from wave instabilities in a one-dimensional neural lattice', Physical Review E 68, 2003, 017201, 1-4.

6. Rinzel, J. and Ermentrout, B. B., 'Analysis of neural excitability and oscillations', in Methods in Neuronal Modeling, C. Koch and I. Segev (eds.), 2nd edn., MIT press, Cambridge, MA, 1998, pp. 251-292.

7. Koch, C., Biophysics of Computation: Information Processing in Single Neurons, Oxford University Press, Oxford, 1998.

8. Ott, E., Grebogi, C., and Yorke, J. A., Physical Review Letters 64, 1990, 1196-1199.

9. Nayfeh, A. H. and Balachandran, B., Applied Nonlinear Dynamics: Analytical,Computational, and Experimental Methods, Wiley-Interscience, New York, 1995.

10. Kazantsev, V. B., 'Selective communication and information processing by excitable systems', Physical Review E 64, 2001, 056210 . 